\title{
Perturbação de Uvarov aplicado à medida clássica de Jacobi
}

\author{
Tatiane Tambarussi \\ Depto de Matemática, Estatística e Computação, FCT, UNESP, \\ 19060-900, Presidente Prudente, SP \\ E-mail: tatitambarussi@hotmail.com \\ Fernando R. Rafaeli \\ Depto de Matemática Aplicada, IBILCE, UNESP, \\ 15054-000, São José do Rio Preto, SP \\ E-mail: rafaeli@ibilce.unesp.br
}

Resumo: $O$ objetivo deste trabalho é apresentar polinômios ortogonais gerados por uma perturbação na medida. O caso que estudaremos é conhecido como medida de Uvarov. Os polinômios gerados pela medida de Uvarov poderam ser escritos através de uma Fórmula de Conexão, que nos permite analisar o comportamento monotônico e assintótico de seus zeros. Por fim aplicaremos estes resultados à medida clássica de Jacobi.

Palavras-chave: Polinômios Ortogonais, Medida, Uvarov.

Neste trabalho vamos apresentar sequências de polinômios ortogonais com respeito a medida $d \phi_{\lambda}(x)=d \phi(x)+\lambda \delta(x-c)$, conhecida como perturbação de Uvarov, onde $d \phi(x)$ é uma medida positiva com suporte finito ou infinito no intervalo $(a, b), \delta(x-c)$ é o funcional Delta de Dirac no ponto $c$, em que $c \notin(a, b)$ e $\lambda$ é um número real não negativo. Logo, os polinômios $p_{n}(\lambda, c ; x)$ que descreveremos são ortogonais com relação ao produto interno

$$
\langle p, q\rangle_{\lambda}=\int_{a}^{b} p(x) q(x) d \phi(x)+\lambda p(c) q(c) .
$$

Para o nosso propósito, introduzimos os polinômios $\left\{p_{n}(x)\right\}_{n \geq 0}$ e $\left\{r_{n}(c ; x)\right\}_{n \geq 0}$ que são ortogonais com relação as medidas $d \phi(x)$ e $d \phi_{2}(x)=(x-c)^{2} d \phi(x)$, respectivamente. Além desses polinômios definimos os polinômios núcleos $K_{n}(x, y)$ por

$$
K_{n}(x, y):=\sum_{k=0}^{n} \frac{P_{k}(x) P_{k}(y)}{\left\|P_{k}\right\|^{2}}
$$

onde $\left\|P_{k}\right\|^{2}=\int_{a}^{b} P_{n}^{2}(x) d \phi(x)$

O teorema abaixo nos mostra uma representação para esta família de polinômios ortogonais, com relação à medida $d \phi_{\lambda}(x)$ apresentando uma forma de escrevê-los, através de uma combinação linear de polinômios.

Teorema 1: [Fórmula de conexão] Os polinômios $\hat{p}_{n}(\lambda, c ; x)$, com a normalização $\hat{p}_{n}(\lambda, c ; x)=$ $\kappa_{n} p_{n}(\lambda, c ; x)$, onde $p_{n}(\lambda, c ; x)$ é mônico, pode ser representado como

$$
\hat{p}_{n}(\lambda, c ; x)=p_{n}(x)+\lambda B_{n}(x-c) r_{n-1}(c ; x),
$$

onde

$$
B_{n}=\frac{-p_{n}(c)}{\left\langle x-c, r_{n-1}\right\rangle_{\phi}}=K_{n-1}(c, c)>0
$$

$e \kappa_{n}=1+\lambda K_{n-1}(c, c)$. 
O teorema a seguir aborda o entrelaçamento dos zeros $\left\{w_{n, k}\right\}_{k=1}^{n}$ dos polinômios $\hat{p}_{n}(\lambda, c, x)$ com $\left\{y_{n, k}\right\}_{k=1}^{n}$ e $\left\{x_{n, k}\right\}_{k=1}^{n}$, zeros de $r_{n}(c ; x)$ e $p_{n}(x)$ respectivamente. Através da representação sugerida pela Fórmula de Conexão, pode-se provar o entrelaçamento dos zeros de $\hat{p}_{n}(\lambda, c ; x)$ com os zeros de $p_{n}(x)$ e $(x-c) r_{n-1}(x)$. Além disso, obtém-se que esses zeros são funções monótonas com relação ao parâmetro $\lambda$ e, quando $\lambda \rightarrow \infty$, os zeros de $\hat{p}_{n}(\lambda, c ; x)$ convergem para os zeros dos polinômios $r_{n-1}(c ; x)$, com velocidade de convergência de ordem $1 / \lambda$.

Teorema 2: Seja $\lambda>0$ e $\left\{y_{n, k}\right\}_{k=1}^{n}$ os zeros dos polinômios $r_{n}(c ; x)$ ortogonais com respeito à medida $d \phi_{2}(x)$.

(i) Se $c \leq a$, então

$$
c<w_{n, 1}<x_{n, 1}<y_{n-1,1}<x_{n, 2}<\cdots<y_{n-1, n-1}<w_{n, n}<x_{n, n} .
$$

Além disso, cada $w_{n, k}$ é uma função decrescente de $\lambda$ e, para cada $k=1,2, \cdots$, $n-1$,

$$
\lim _{\lambda \rightarrow \infty} w_{n, 1}=c, \quad \lim _{\lambda \rightarrow \infty} w_{n, k+1}=y_{n-1, k}
$$

bem como

$$
\begin{aligned}
\lim _{\lambda \rightarrow \infty} \lambda\left[w_{n, 1}-c\right] & =\frac{-p_{n}(c)}{K_{n-1}(c, c) r_{n-1}(c)} \\
\lim _{\lambda \rightarrow \infty} \lambda\left[w_{n, k+1}-y_{n-1, k}\right] & =\frac{-p_{n}\left(y_{n-1, k}\right)}{K_{n-1}(c, c)\left(y_{n-1, k}-c\right) r_{n-1}^{\prime}\left(y_{n-1, k}\right)} .
\end{aligned}
$$

(ii) Se $c \geq b$, então

$$
x_{n, 1}<w_{n, 1}<y_{n-1,1}<\cdots<x_{n, n-1}<w_{n, n-1}<y_{n-1, n-1}<x_{n, n}<w_{n, n}<c .
$$

Além disso, cada $w_{n, k}$ é uma função crescente de $\lambda$ e, para cada $k=1,2, \cdots, n-1$,

$$
\lim _{\lambda \rightarrow \infty} w_{n, n}=c, \lim _{\lambda \rightarrow \infty} w_{n, k}=y_{n-1, k},
$$

bem como

$$
\begin{aligned}
\lim _{\lambda \rightarrow \infty} \lambda\left[c-w_{n, n}\right] & =\frac{p_{n}(c)}{K_{n-1}(c, c) r_{n-1}(c)} \\
\lim _{\lambda \rightarrow \infty} \lambda\left[y_{n-1, k}-w_{n, k}\right] & =\frac{p_{n}\left(y_{n-1, k}\right)}{K_{n-1}(c, c)\left(y_{n-1, k}-c\right) r_{n-1}^{\prime}\left(y_{n-1, k}\right)} .
\end{aligned}
$$

Vamos considerar alguns casos particulares da perturbação de Uvarov. No artigo de Huertas, Marcellán e Rafaeli [5] foi proposto a modificação da medida clássica de Jacobi através da perturbação de Uvarov.

A sequência de polinômios mônicos de Jacobi $\left\{p_{n}^{(\alpha, \beta)}(x)\right\}$, é ortogonal com relação á medida $d \phi_{\alpha, \beta}(x)=(1-x)^{\alpha}(1+x)^{\beta} d x$. Consideremos as seguintes perturbações na medida $d \phi_{\alpha, \beta}(x)$ :

$$
\begin{aligned}
d \phi(\lambda, 1 ; x) & =d \phi_{\alpha, \beta}(x)+\lambda \delta(x-1), \\
d \phi(\lambda,-1 ; x) & =d \phi_{\alpha, \beta}(x)+\lambda \delta(x+1) .
\end{aligned}
$$

A partir, das medidas introduzidas em (10) e (11), obtemos duas novas famílias de polinômios ortogonais mônicos as quais, denotaremos por $\left\{p_{n}^{(\alpha, \beta)}(\lambda, 1 ; x)\right\}$ e $\left\{p_{n}^{(\alpha, \beta)}(\lambda,-1 ; x)\right\}$, respectivamente. Esses polinômios são conhecidos na literatura como polinômios ortogonais do tipo Jacobi ou polinômios ortogonais de Jacobi-Koornwinder. De fato eles apareceram pela primeira vez no trabalho de Koornwinder [8]. 
Com a normalização $\hat{p}_{n}^{(\alpha, \beta)}(\lambda, c ; x)=\kappa_{n} p_{n}^{(\alpha, \beta)}(\lambda, c ; x)$ introduzida no Teorema 1 , onde $\kappa_{n}=$ $1+\lambda K_{n-1}(c, c)$ e $p_{n}^{(\alpha, \beta)}(\lambda, c ; x)$ é um polinômio do tipo Jacobi mônico, obtemos a Fórmula de Conexão para os polinômios $\hat{p}_{n}^{(\alpha, \beta)}(\lambda, 1 ; x)$ :

$$
\hat{p}_{n}^{(\alpha, \beta)}(\lambda, 1 ; x)=p_{n}^{(\alpha, \beta)}(x)+\lambda K_{n-1}(1,1)(x-1) p_{n-1}^{(\alpha+2, \beta)}(x) .
$$

Na equação (12), temos um polinômio tipo Jacobi escrito como combinação linear de um polinômio ortogonal com relação à medida $d \phi_{\alpha, \beta}(x)$, e outro com relação à medida $d \phi_{2}(x)$ resultando em um polinômio tipo Jacobi deslocado no parâmetro $\alpha$, com isso podemos utilizar o Teorema 2, onde basta provar que os zeros desses polinômios se entrelaçam, e teremos hipóteses suficientes para analisar o comportamento dos zeros dos polinômios tipo Jacobi com relação a monotonicidade e assintoticidade.

Teorema 3: Sejam $\alpha, \beta>-1$ e sejam $x_{n, k}(\alpha, \beta)$ e $x_{n, k}(\alpha, \beta ; \lambda):=x_{n, k}(\alpha, \beta ; 1, \lambda), k=1, \cdots, n$, os zeros de $p_{n}^{(\alpha, \beta)}(x)$ e $p_{n}^{(\alpha, \beta)}(\lambda, 1 ; x)$, respectivamente. Então vale a seguinte propriedade de entrelaçamento:

$$
\begin{aligned}
& x_{n, 1}(\alpha, \beta)<x_{n, 1}(\alpha, \beta ; \lambda)<x_{n-1,1}(\alpha+2, \beta)<x_{n, 2}(\alpha, \beta)<x_{n, 2}(\alpha, \beta ; \lambda)<\cdots< \\
& <x_{n, n-1}(\alpha, \beta)<x_{n, n-1}(\alpha, \beta ; \lambda)<x_{n-1, n-1}(\alpha+2, \beta)<x_{n, n}(\alpha, \beta)<x_{n, n}(\alpha, \beta ; \lambda)<1,
\end{aligned}
$$

Além disso, cada $x_{n, k}(\alpha, \beta ; \lambda)$ é uma função crescente de $\lambda$ e, para $k=1, \cdots, n-1$,

$$
\lim _{\lambda \rightarrow \infty} x_{n, n}(\alpha, \beta ; \lambda)=1, \quad \lim _{\lambda \rightarrow \infty} x_{n, k}(\alpha, \beta ; \lambda)=x_{n-1, k}(\alpha+2, \beta),
$$

$e$,

$$
\begin{aligned}
& \lim _{\lambda \rightarrow \infty} \lambda\left[1-x_{n, n}(\alpha, \beta ; \lambda)\right]=g_{n}(\alpha, \beta), \\
& \lim _{\lambda \rightarrow \infty} \lambda\left[x_{n-1, k}(\alpha+2, \beta)-x_{n, k}(\alpha, \beta ; \lambda)\right]=\frac{\left[1+x_{n-1, k}(\alpha+2, \beta)\right] g_{n}(\alpha, \beta)}{2(\alpha+2)},
\end{aligned}
$$

onde

$$
g_{n}(\alpha, \beta)=\frac{2^{\alpha+\beta+2} \Gamma(n) \Gamma(\alpha+2) \Gamma(\alpha+3) \Gamma(n+\beta)}{\Gamma(n+\alpha+2) \Gamma(n+\alpha+\beta+2)} .
$$

Para ilustrar o resultado do Teorema observamos o gráfico do polinômio $\hat{p}_{3}^{(1,1)}(\lambda+\epsilon, 1 ; x)$, fixando $\lambda=0$ para alguns valores de $\epsilon>0$, observamos a monotonicidade dos zeros de $\hat{p}_{3}^{(1,1)}(\lambda+\epsilon, 1 ; x)$ com relação a $\lambda$.

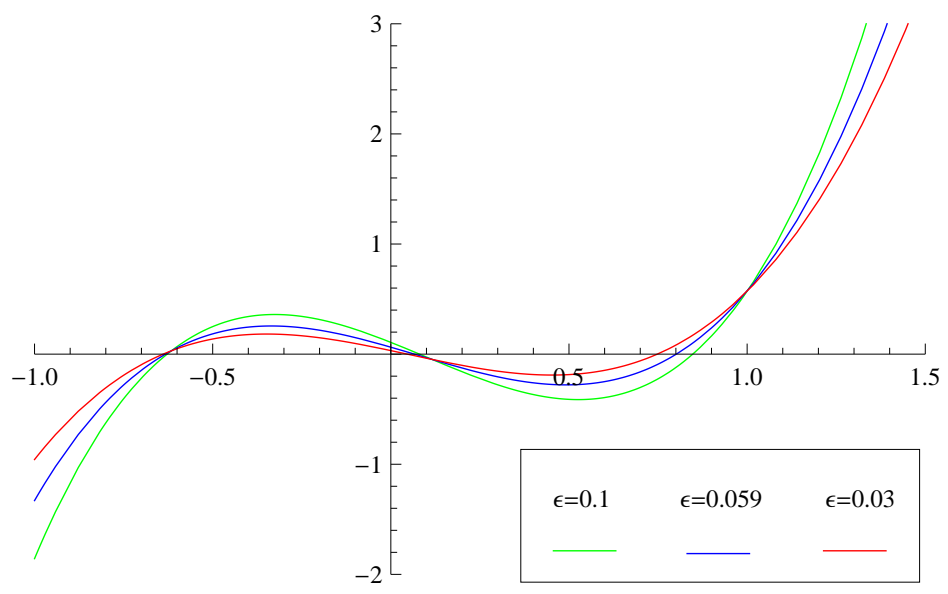

Figura 1: Gráfico de $\hat{p}_{3}^{(1,1)}(\lambda+\epsilon, 1 ; x)$ 
Na Tabela 1 apresentamos os zeros de $\hat{p}_{3}^{(1,1)}(\lambda, 1 ; x)$ para alguns valores de $\lambda$. Notamos que todos os zeros desse polinômio crescem quando $\lambda$ cresce, além disso, observamos que o último zero converge para 1 e os outros convergem para os zeros de $\hat{p}_{2}^{(3,1)}(x)$ que são $x_{2,1}=-0.615963$ e $x_{2,2}=0.115963$.

\begin{tabular}{llll}
\hline \hline$\lambda$ & $x_{3,1}(1,1, \lambda)$ & $x_{3,2}(1,1, \lambda)$ & $x_{3,3}(1,1, \lambda)$ \\
\hline \hline 0 & $-0,654654$ & 0 & 0,654654 \\
1 & $-0,617598$ & 0,111189 & 0,975159 \\
10 & $-0,616132$ & 0,115468 & 0,997353 \\
100 & $-0,61598$ & 0,115913 & 0,999734 \\
1000 & $-0,615964$ & 0,115958 & 0,999973 \\
\hline \hline
\end{tabular}

Tabela 1: Zeros de $\hat{p}_{3}^{(1,1)}(\lambda, 1 ; x)$

Os mesmos resultados podem ser observados quando $c=-1$. Neste caso a Fórmula de Conexão para $\hat{p}_{n}^{(\alpha, \beta)}(\lambda,-1 ; x)$ é dada por

$$
\hat{p}_{n}^{(\alpha, \beta)}(\lambda,-1 ; x)=p_{n}^{(\alpha, \beta)}(x)+\lambda K_{n-1}(-1,-1)(x+1) p_{n-1}^{(\alpha, \beta+2)}(x) .
$$

Através desta representação, pode-se provar o entrelaçamento dos zeros $\hat{p}_{n}^{(\alpha, \beta)}(\lambda,-1 ; x)$ com $p_{n}^{(\alpha, \beta)}(x)$ e $(x+1) p_{n-1}^{(\alpha, \beta+2)}(x)$, podendo assim estudar o comportamento de seus zeros com relação ao parâmetro $\lambda$, como mostra o teorema abaixo.

Teorema 4: Sejam $\alpha, \beta>-1$ e sejam $x_{n, k}(\alpha, \beta)$ e $x_{n, k}(\alpha, \beta ; \lambda):=x_{n, k}(\alpha, \beta ;-1, \lambda), k=$ $1, \cdots, n$, os zeros de $p_{n}^{(\alpha, \beta)}(x)$ e $p_{n}^{(\alpha, \beta)}(\lambda,-1 ; x)$, respectivamente. Então vale a seguinte propriedade de entrelaçamento:

$$
\begin{aligned}
-1<x_{n, 1}(\alpha, \beta ; \lambda)<x_{n, 1}(\alpha, \beta)< & x_{n-1,1}(\alpha, \beta+2)<x_{n, 2}(\alpha, \beta ; \lambda)<x_{n, 2}(\alpha, \beta)<\cdots \\
& <x_{n-1, n-1}(\alpha, \beta+2)<x_{n, n}(\alpha, \beta ; \lambda)<x_{n, n}(\alpha, \beta),
\end{aligned}
$$

Além disso, cada $x_{n, k}(\alpha, \beta ; \lambda)$ é uma função decrescente de $\lambda$ e, para $k=1, \cdots, n-1$,

$$
\lim _{\lambda \rightarrow \infty} x_{n, 1}(\alpha, \beta ; \lambda)=-1, \quad \lim _{\lambda \rightarrow \infty} x_{n, k+1}(\alpha, \beta ; \lambda)=x_{n-1, k}(\alpha, \beta+2),
$$

$e$,

$$
\begin{aligned}
& \lim _{\lambda \rightarrow \infty} \lambda\left[x_{n, 1}(\alpha, \beta ; \lambda)+1\right]=h_{n}(\alpha, \beta) \\
& \lim _{\lambda \rightarrow \infty} \lambda\left[x_{n, k+1}(\alpha, \beta ; \lambda)-x_{n-1, k}(\alpha, \beta+2)\right]=\frac{\left[1-x_{n-1, k}(\alpha, \beta+2)\right] h_{n}(\alpha, \beta)}{2(\beta+2)},
\end{aligned}
$$

onde

$$
h_{n}(\alpha, \beta)=\frac{2^{\alpha+\beta+2} \Gamma(n) \Gamma(\beta+2) \Gamma(\beta+3) \Gamma(n+\alpha)}{\Gamma(n+\beta+2) \Gamma(n+\alpha+\beta+2)} .
$$

Para ilustrar o resultado do Teorema 4 , o gráfico do polinômio $\hat{p}_{3}^{(0,3)}(\lambda+\epsilon,-1 ; x)$, fixando $\lambda=0$ e para valores de $\epsilon>0$, notamos o comportamento monótono dos zeros de $\hat{p}_{3}^{(0,3)}(\lambda+\epsilon, 1 ; x)$ com relação a $\lambda$. 


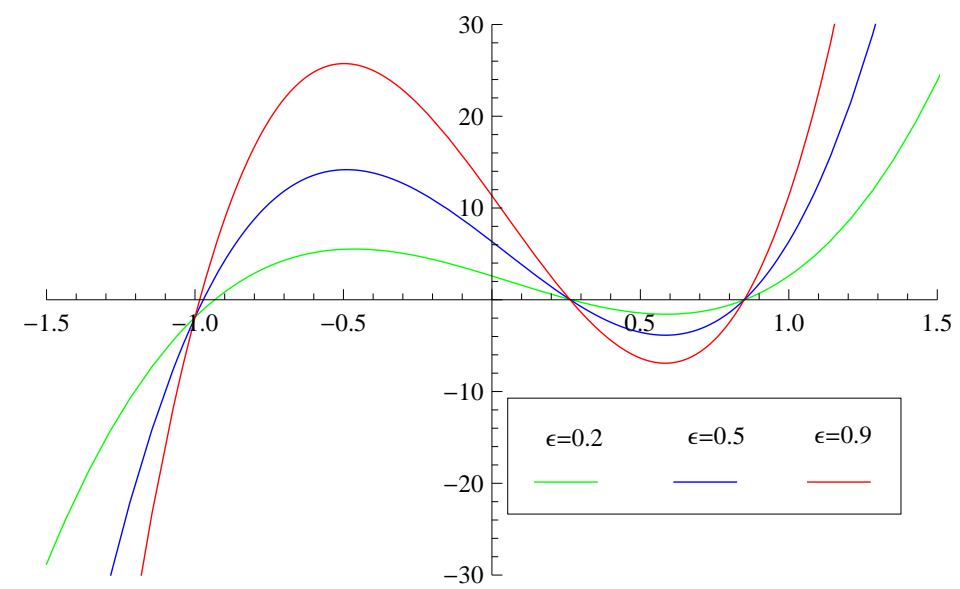

Figura 2: Gráfico de $\hat{p}_{3}^{(0,3)}(\lambda+\epsilon,-1 ; x)$.

Na Tabela 2 , apresentamos os zeros de $\hat{p}_{3}^{(0,3)}(\lambda,-1 ; x)$ para alguns valores de $\lambda$. Notamos que todos os zeros desse polinômio decrescem quando $\lambda$ cresce, além disso, observamos que o primeiro zero converge para -1 , e os outros convergem para os zeros de $\hat{p}_{2}^{(0,5)}(x)$ que são $x_{2,1}=-0.261583$ e $x_{2,2}=0.849528$.

\begin{tabular}{llll}
\hline \hline$\lambda$ & $x_{3,1}(0,3, \lambda)$ & $x_{3,2}(0,3, \lambda)$ & $x_{3,3}(0,3, \lambda)$ \\
\hline \hline 0 & $-0,273471$ & 0,397623 & 0,875848 \\
1 & $-0,985755$ & 0,262648 & 0,849745 \\
10 & $-0,998551$ & 0,26169 & 0,84955 \\
100 & $-0,99985$ & 0,261594 & 0,84953 \\
1000 & $-0,999985$ & 0,261584 & 0,849528 \\
\hline \hline
\end{tabular}

Tabela 2: Zeros de $\hat{p}_{3}^{(0,3)}(\lambda,-1 ; x)$

\section{Referências}

[1] BRACCIALI, C. F., Dimitrov, D. K., RANGA, A. S. "Chain sequences and symmetric generalized orthogonal polynomials", J. Comput. Appl. Math. 143 (2002) 95-106.

[2] CHIHARA, T. S., "An Introduction to Orthogonal Polynomials", Mathematics and its Applications Series, Gordon and Breach, New York, 1978.

[3] Dimitrov, D. K., MARCEllán, F., RAFAeli, F. R. "Monotonicity of zeros of Laguerre-Sobolev type orthogonal polynomials", J. Math. Anal. Appl. (2010) 80-89.

[4] Dimitrov, D. K., Mello, M. V., RAFAELi, F. R. "Monotonicity of zeros of Jacobi-Sobolev type orthogonal polynomials", J. Comput. Appl. Numer. Math. (2010) 263276.

[5] HUERTAS,E. J., MARCELLÁN, F., RAFAELI, F.R., "Zeros of orthogonal polynomials generated by canonical pertubations of measures", J. Comput. Appl. Math. 218 (2012) 7109-7127.

[6] HUERTAS,E. J., "Analytic Properties of Krall-type and Sobolev-type Orthogonal Polynomials", Ph.D. Thesis, Universidad Carlos III de Madrid, Leganés, 2012. 
[7] ISMAIL, M. E. H., "Classical and Quantum Orthogonal Polynomials in one Variable", Vol. 98 of Encyclopedia of Mathematics and its Applications. Cambridge University Press, Cambridge, 2005. With two chapters by Walter Van Assche, With a foreword by Richard A. Askey.

[8] KOORNWINDER, T. H. "Orthogonal polynomials with weight function $(1-x)^{\alpha}(1+x)^{\beta}+M \delta(x+1)+N \delta(x-1) "$, Canad. Math. Bull. 27 (1984) 205-214.

[9] OBRECHKOFF, N., "Zeros of Polynomials", Acad. G. Bonchev, Bulgaria, Sofia, 2003.

[10] SZEGÖ, G., "Orthogonal polynomials", American Math. Society, Colloq. Publ., New York, $23,1939$. 